\title{
Generation of knock-in cynomolgus monkey via CRISPR/Cas9 editing
}

Cell Research (2018) 28:379-382. doi:10.1038/cr.2018.9; published online 12 January 2018

\section{Dear Editor,}

Genetically modified monkey models that exhibit human disease symptoms are versatile tools for studying human disease mechanisms and advancing therapeutic treatments [1]. However, generation of gene-targeted monkeys remains very challenging, due to the long breeding cycle and low gene-editing efficiency $[1,2]$. The CRISPR/Cas9 system has been used to generate gene-targeted animals in many species by directly injecting Cas9 mRNA and single-guide RNAs (sgRNAs) into pronuclear stage zygotes [3]. Several recent studies have shown that the CRISPR/Cas9 system could also be used to generate gene-knockout monkeys [4-10]. However, no gene knock-in monkey has been reported, presumably because of the low knock-in rate [2].

We previously reported that homology-mediated end joining (HMEJ)-based method could efficiently achieve targeted integration in the embryos of cynomolgus monkeys [11]. However, the low blastocyst rate of the injected embryos $(26.7 \%, 4$ out of 15$)$ may hinder the generation of live-birth monkeys [11]. We thus further optimized the zygote injection conditions, including the concentration of donor plasmids and the total injection volume, to improve the knock-in efficiency while maintaining the normal embryonic development. In this study, we aimed to insert Actb (intron 4 to exon 5)-p2A-mCherry into the intron 4 of the Actb gene (highly expressed in most cells/tissues) to achieve mCherry expression under the control of the Actb promoter (Supplementary information, Figure S1A). The knock-in efficiencies were evaluated by mCherry fluorescence signals in blastocysts (Supplementary information, Figure S1B). By co-injection of different volumes ( 4-10 pL) and concentrations of donor plasmid (50-100 $\mathrm{ng} / \mu \mathrm{L})$ together with Cas9 mRNA $(100 \mathrm{ng} / \mu \mathrm{L})$ and sgRNA $(50 \mathrm{ng} / \mu \mathrm{L})$ into fertilized monkey oocytes, we found that $\sim 4 \mathrm{pL}$ of total volume and $100 \mathrm{ng} / \mu \mathrm{L}$ of donor plasmid could achieve both high knock-in efficiency (5 out of 6 ) and high blastocyst rate (6 out of 11; Supplementary information, Figure S1C and S1D).
For embryo transplantation experiments, we fertilized the monkey oocytes with intracytoplasmic sperm injection (ICSI), and $6 \mathrm{~h}$ later injected a mixture of Cas9 mRNA (100 ng/ $\mu \mathrm{L})$, sgRNA (50 $\mathrm{ng} / \mu \mathrm{L})$ and HMEJ donor plasmid $(100 \mathrm{ng} / \mu \mathrm{L})$ with a total injection volume of $\sim 4 \mathrm{pL}$. We first transferred 27 injected zygotes into 9 surrogate females, but found no pregnancy. To achieve a higher rate of pregnancy, we reduced the injection volume to $\sim 2 \mathrm{pL}$. A total of 42 injected zygotes were transferred into 12 surrogate females. Of the recipient mothers, five pregnancies were established and one miscarriage occurred 80 days after embryo transfer (Supplementary information, Table S1). The rest four pregnancies included one twin and three singletons. Successful delivery at full term (147-163 days) yielded a pair of twin babies (\#1 male and \#2 female) and three female singletons (\#3, \#4 and \#5). One of the twin babies (\#2) died one day after birth and \#3 died on the 13th day after birth due to enteritis (Supplementary information, Table S1). Three other monkeys survived and exhibited apparently normal growth with time. Direct epifluorescence of the toes of newborn babies showed that four monkeys (\#1, $\# 2$, \#3 and \#5) exhibited visible mCherry fluorescence as compared to wild-type newborn monkeys (Figure 1A).

Next, ear tissue sections were obtained from five monkeys and mCherry expression was observed in all monkeys (Figure 1B). We also obtained tissue sections from the tail, toe, gut, muscle, heart, kidney, brain and ovary of the two deceased female monkeys (\#2, \#3). We observed mosaic expression of mCherry in all sections (10\%-90\% of cells, Figure 1B and Supplementary information, Figure S2A and S2B). Notably, we detected MVH (a germ cell marker) expression in mCherry $^{+}$cells of the ovaries of monkeys \#2 and \#3 (Supplementary information, Figure S2C), suggesting the potential capability of germline transmission of the knock-in monkeys. In addition, mCherry expression was confirmed by western blotting (Supplementary information, Figure S2D). All these samples from monkeys \#2 and \#3 and ears of three live monkeys (\#1, \#4, \#5) were also used for PCRbased genotyping. Targeted integration was found in 
A

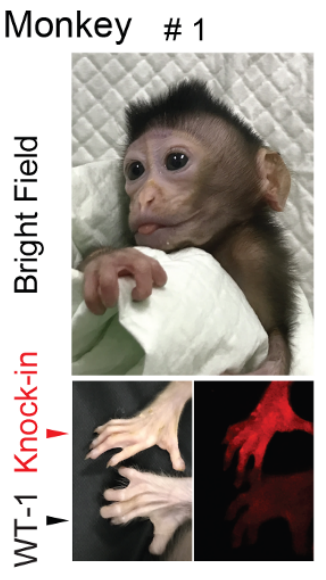

B

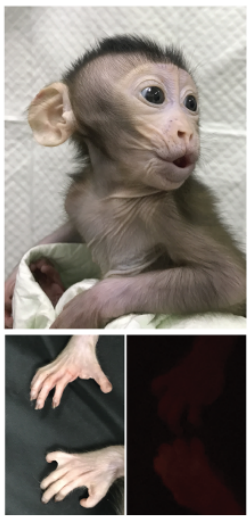

\# 5

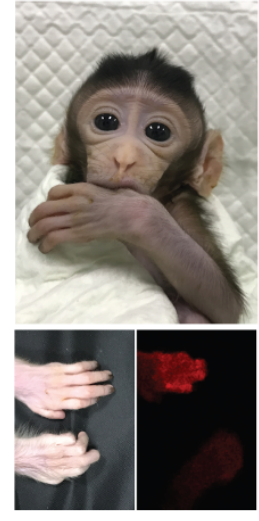

\# 3 WT-2 \#2
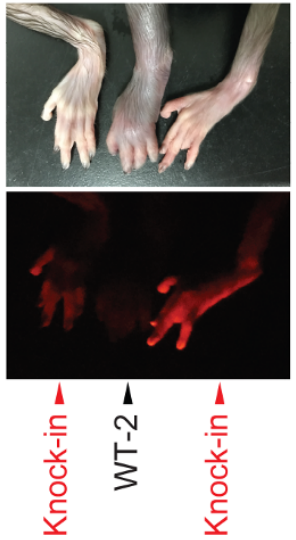

WT $\# 1$

Ear
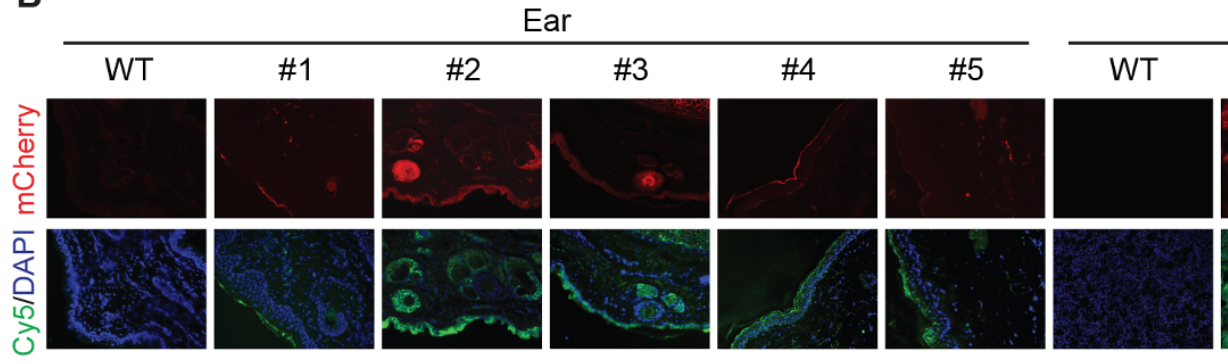

Ovary

C

Monkey tissues

\#2

\#3

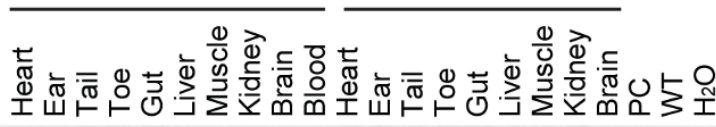

5 ' junction

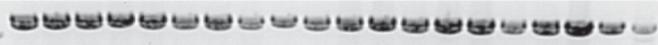

3' junction

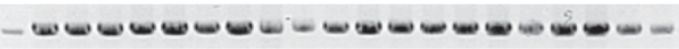

Actb
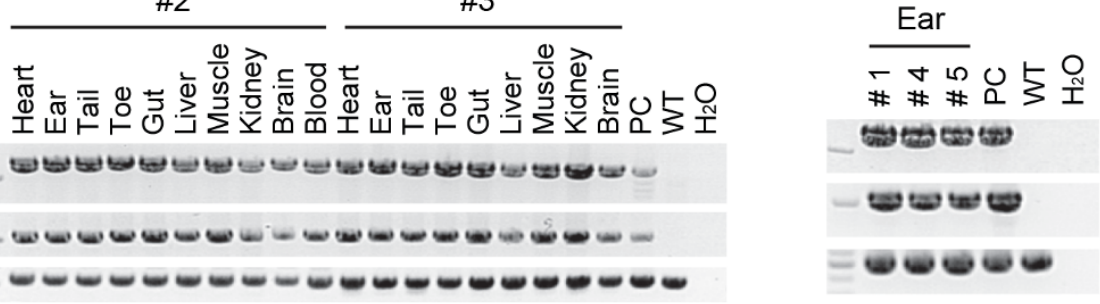

D

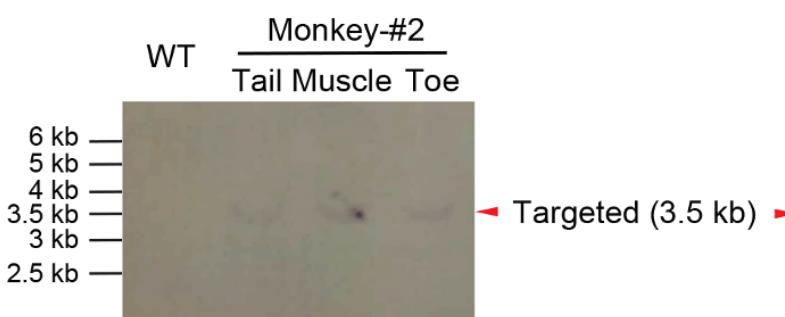

WT $\frac{\text { Monkey-\#3 }}{\text { Tail Muscle Toe }}$

Ndel-digested

Internal probe

$3 \mathrm{~kb}$

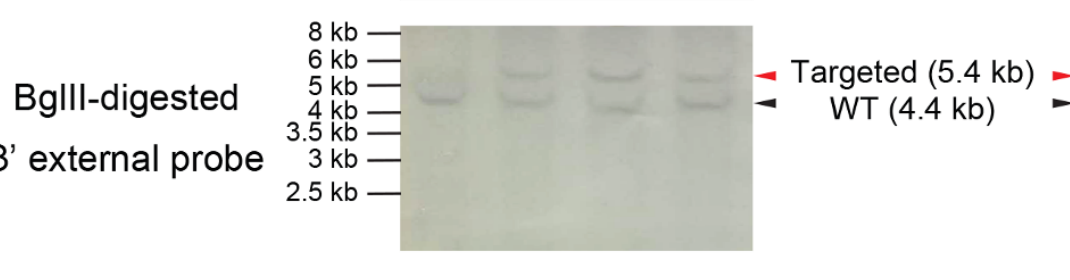

E

\begin{tabular}{ccccccccc}
\hline $\begin{array}{c}\text { Injected Embryos } \\
\text { (Oocyte donor) }\end{array}$ & $\begin{array}{c}\text { Surrogate } \\
\text { recipients }\end{array}$ & $\begin{array}{c}\text { Pregnancy } \\
\text { recipients }\end{array}$ & Newborns & Survival & $\begin{array}{c}\mathrm{EF} \\
\text { mCherry }\end{array}$ & $\begin{array}{c}\text { IF } \\
\text { mCherry }\end{array}$ & PCR & Southern \\
\hline $42(2)$ & 12 & $5^{\mathrm{a}}$ & 5 & $3(5)^{\mathrm{b}}$ & $4(5)^{\mathrm{b}}$ & $5(5)^{\mathrm{b}}$ & $5(5)^{\mathrm{b}}$ & $2(2)^{\mathrm{b}}$ \\
\hline
\end{tabular}


Figure 1 Actb-p2A-mCherry knock-in monkeys generated by HMEJ-based method. (A) The knock-in cynomolgus monkey infants. Left top panel, images of three survived newborns (\#1, \#4 and \#5); left bottom panel, epifluorescent images of the toes of three survived newborns (\#1, \#4 and \#5); right panel, epifluorescent images of the toes of two deceased newborns (\#2 and \#3). Red arrowheads indicate the toes of knock-in monkeys, while black arrowheads indicate the toes of wild-type monkeys. Images were collected at the different ages of monkeys: \#1, \#4 and \#5, 1 month; \#2, day 1; \#3, day 13; WT-1, 1 month; WT-2, day 1. All monkeys except \#4 showed mCherry epifluorescence in their toes. (B) Immunofluorescence analysis using an anti-mCherry antibody of ear tissues from all the newborns and ovary tissues from two deceased monkeys. Blue, DAPI; green, Cy5; red, mCherry. Scale bar, $100 \mu \mathrm{m}$. (C) Genotyping analysis of the newborns. PCR products amplified from 5' and 3' junction sites of DNA samples of the newborns. PC, positive control from COS-7 cells with Actb-p2A-mCherry knock-in. WT, genomic DNA from wild-type monkey. (D) Southern blot analysis. Ndel-digested and Bg/ll-digested genomic DNAs from monkeys \#2 and \#3 were hybridized with internal mCherry probe and 3' external probe, respectively. Expected fragment sizes: $\mathrm{WT}=\mathrm{N} / \mathrm{A}$, Targeted $=3.5 \mathrm{~kb}$ (internal mCherry probe); WT $=4.4 \mathrm{~kb}$, Targeted $=5.4 \mathrm{~kb}$ (external probe). (E) Summary of Actb-p2A-mCherry knock-in monkeys generated by HMEJ-mediated targeted integration. Cas9 mRNA (100 ng/ $\mu \mathrm{L})$, sgRNAs $(50 \mathrm{ng} / \mu \mathrm{L})$, and donor vectors $(100 \mathrm{ng} / \mu \mathrm{L})$ were injected into fertilized eggs, with a total injection volume of $\sim 2 \mathrm{pL}$. EF, epifluorescence; IF, immunofluorescence. ${ }^{a}$ One carried twins and one miscarriage occurred 80 days after embryo transfer. ${ }^{b}$ Number in the brackets indicates total samples analyzed.

all samples (Figure 1C), and the precise in-frame integrations occurred at $5^{\prime}$ and $3^{\prime}$ junctions (Supplementary information, Figure S3). We also detected NHEJ-induced mutations at Actb locus in all the monkeys (Supplementary information, Figure S4). Moreover, for monkey \#2, we used a set of primers to amplify the region across the transgene and its adjacent $5^{\prime}$ and $3^{\prime}$ arms by PCR (Supplementary information, Figure S5A). Sequencing of these PCR products confirmed the correct transgene integration and the absence of mutation in the knock-in alleles (Supplementary information, Figure S5B). Furthermore, Southern blot analysis on monkeys \#2 and \#3 confirmed the correct transgene integration (Figure 1D) and the absence of no additional randomly integrated transgene (Figure 1D, internal probe). To study off-target effects, we examined the 12 highest-ranked predicted off-target sites in five knock-in monkeys using ear tissues and found that no mutation occurred at any of these loci (Supplementary information, Figure S6). Taken together, we have successfully achieved CRISPR/Cas9-mediated targeted transgene integration using HMEJ strategy in monkey genome by one-cell embryo microinjection.

In summary, we found that HMEJ-based knock-in strategy represents an efficient and simple method for generating targeted genetic modifications in monkeys (Figure 1E). However, knock-in monkeys generated by this approach showed mosaicism. Therefore, further serial crossbreeding is required to generate complete gene knock-in monkeys. Such a procedure is very laborious due to a long breeding cycle (5-6 years) and a small litter size (1 per birth) in macaque monkeys. Injection of Cas 9 protein instead of mRNA at earlier embryonic stage may achieve transgenic knock-in at one-cell stage without mosaicism [12]. Alternatively, combination of testicular xenografts [13] with our HMEJ-based knock-in approach could produce a large number of F1 knock-in monkeys in only three years, and thus accelerate the generation of human disease models. The knock-in approach shown here, even with mosaicism, might be useful for generating tool monkeys that express exogenous proteins in specific cell types for optogenetic [14] and pharmocogenetic [15] manipulations.

Materials and Methods are available in Supplementary information, Data S1 and Table S2.

\section{Acknowledgments}

We thank Drs Mu-ming Poo (Institute of Neuroscience, CAS) and Jiazhi Hu (Peking University) for helpful discussions and insightful comments on this manuscript. This work was supported by the National Science and Technology Major Project (2017YFC1001302), CAS Strategic Priority Research Program (XDB02050007 and XDA01010409), the National High-tech R\&D Program (863 Program; 2015AA020307), the National Natural Science Foundation of China (31522037 and 31500825), China Youth Thousand Talents Program to HY; Break-through project of Chinese Academy of Sciences (HY and QS), Shanghai Sailing Plan for the Young Scientific Talents (15YF1414700 to LS), The National Key Technology R\&D Program of China (2014BAI03B00 to QS), Shanghai City Committee of Science and Technology (16JC1420202 to HY; 14140900100 to QS), CAS Hundreds of Talents Program (QS).

Xuan Yao ${ }^{1, *}$, Zhen Liu ${ }^{1, *}$, Xing Wang ${ }^{1,2, *}$, Yan Wang ${ }^{1}$, Yan-Hong $\mathrm{Ni}^{1}$, Liang $\mathrm{Lai}^{3}$, Ruilin Sun ${ }^{3}$, Linyu Shi ${ }^{1}$, Qiang Sun ${ }^{1}$, Hui Yang ${ }^{1}$

${ }^{I}$ Institute of Neuroscience, State Key Laboratory of Neuroscience, Key Laboratory of Primate Neurobiology, CAS Center for Excellence in Brain Science and Intelligence Technology, Shanghai Institutes for Biological Sciences, Chinese Academy of Sciences, Shanghai 200031, China; ${ }^{2}$ College of Life Sciences, University of Chinese Academy of Sciences, Beijing 100049, China; ${ }^{3}$ Shanghai Model Organisms Center, Inc., Shanghai, China *These three authors contributed equally to this work.

Correspondence: Qiang Sun ${ }^{\mathrm{a}}$, Hui Yang ${ }^{\mathrm{b}}$

${ }^{\mathrm{a}}$ E-mail: qsun@ion.ac.cn

${ }^{\mathrm{b}} \mathrm{E}-\mathrm{mail}$ : huiyang@ion.ac.cn

\section{References}

1 Izpisua Belmonte JC, Callaway EM, Caddick SJ, et al. Neuron 2015; 
86:617-631.

Guo X, Li XJ. Cell Res 2015; 25:767-768.

Wang H, Yang H, Shivalila CS, et al. Cell 2013; 153:910-918.

Niu Y, Shen B, Cui Y, et al. Cell 2014; 156:836-843.

Wan H, Feng C, Teng F, et al. Cell Res 2015; 25:258-261.

Chen Y, Zheng Y, Kang Y, et al. Hum Mol Genet 2015; 24:3764-3774.

Kang Y, Zheng B, Shen B, et al. Hum Mol Genet 2015; 24:7255-7264.

Chen Y, Cui Y, Shen B, et al. Cell Res 2015; 25:262-265.

9 Zhao H, Tu Z, Xu H, et al. Cell Res 2017; 27:1293-1297.
10 Zuo E, Cai YJ, Li K, et al. Cell Res 2017; 27:933-945.

11 Yao X, Wang X, Hu X, et al. Cell Res 2017; 27:801-814.

12 Ma H, Marti-Gutierrez N, Park SW, et al. Nature 2017; 548:413-419.

13 Liu Z, Nie YH, Zhang CC, et al. Cell Res 2016; 26:139-142.

14 Galvan A, Caiola MJ, Albaugh DL. J Neural Transm (Vienna) 2017 Feb 25. doi:10.1007/s00702-017-1697-8

15 Heilig M, Goldman D, Berrettini W, et al. Nat Rev Neurosci 2011; 12:670-684.

(Supplementary information is linked to the online version of the paper on the Cell Research website.) 